
\title{
25 Research Soure \\ Investigating Resistance to Emamectin Benzoate in the Tomato Borer Tuta Absoluta
}

\section{Emmanouil Roditakis ( $\nabla$ eroditakis@hmu.gr)}

Elleniko Mesogeiako Panepistemio https://orcid.org/0000-0002-5938-2977

\section{Marianna Stavrakaki}

Hellenic Mediterranean University: Elleniko Mesogeiako Panepistemio

Aris llias

Foundation of Research and Technology Hellas: Idryma Technologias kai Ereunas

\section{Panagiotis loannidis}

Foundation of Research and Technology Hellas: Idryma Technologias kai Ereunas

\section{John Vontas}

Foundation of Research and Technology Hellas: Idryma Technologias kai Ereunas

\section{Research Article}

Keywords: Tuta absoluta, resistance, avermectins, emamectin benzoate, abamectin; tomato, borer, P450s, Greece

Posted Date: August 23rd, 2021

DOI: https://doi.org/10.21203/rs.3.rs-816356/v1

License: (c) (i) This work is licensed under a Creative Commons Attribution 4.0 International License. Read Full License 


\section{Abstract}

The tomato borer Tuta absoluta is a major pest of tomato mainly controlled by chemical insecticides. However, development of resistance to specific chemical classes has made control of the pest extremely difficult. Emamectin benzoate belongs to the avermectin mode of action and to date, low or no resistance levels against this insecticide have been documented. Recently, reduced efficacy of emamectin benzoate was documented, in a field population from Crete (9-fold resistant ratio (RR)). Subsequent laboratory selections with emamectin benzoate for eight sequential generations, resulted in an increase of the RR to 60 -fold, the highest resistance level reported to the particular insecticide. Hereby, we are presenting the characterization of emamectin benzoate resistance in $T$. absoluta. Sequencing of the $\mathrm{GluCl}$ and GABA receptor $(\mathrm{rdl})$ genes, the molecular targets of emamectin benzoate, indicted absence of non-synonymous SNPs. The use of known enzyme inhibitors (PBO, DEF and DEM) revealed that P450s partially synergized emamectin benzoate resistance, suggesting potential implication of metabolic resistance. RNA-seq approach was used to identify differentially expressed genes, from emamectin benzoate resistant and susceptible $T$. absoluta populations. Twelve libraries were sequenced using the lllumina platform, which generated $81 \mathrm{Gbp}$, thus substantially increasing the number of publicly available genomic resources for this species. The de novo transcriptome assembly consisted of 549,601 contigs, grouped in 233,453 unigenes. Differential expression analysis and QPCR validation revealed over-expression of one unigene similar to cytochrome P450 (Clan 4) potentially implicated in emamectin benzoate resistance, supporting further the involvement of P450s in the observed resistance phenotype.

\section{Key Points:}

- Low resistance to avermectins was reported in Tuta absoluta

- Laboratory selection with emamectin benzoate resulted in high resistance levels in the selected strain (60-fold)

- PBO synergised the emamectin benzoate activity, supporting further the potential involvement of P450s

- Differential expression analysis revealed over-expression of one cytochrome P450 enzyme

\section{Introduction}

The tomato leaf miner Tuta absoluta (Meyrick) (Lepidoptera: Gelechiidae) is a multivoltine, invasive moth originating from South America. It is considered as a major pest of tomato (Lycopersicon esculentum Mill.). The tomato leaf miner invaded Spain in 2006 (Urbaneja et al. 2007) and since it has rapidly spread in most tomato-growing regions of the world such as Europe, Africa, Middle East and Asia, threatening tomato production globally in both greenhouse and open field crops (Desneux et al. 2010; Desneux et al. 2011).

The tomato leaf miner management relies heavily on applications with chemical insecticides (Siqueira et al. 2000b; Roditakis et al. 2013) however, reports of control failure have clearly illustrated the potential of 
this pest to develop resistance to multiple classes of insecticide (Siqueira et al. 2000b; Siqueira et al. 2001; Silva et al. 2011; Roditakis et al. 2015; Silva et al. 2016a; Silva et al. 2016b; Haddi et al. 2017). The most extensively used insecticides belong to four distinct chemical classes addressing different modes of action, namely the diamides, the avermectins, the spinosyns and the oxadiazines (Sparks and Nauen 2015).

Avermectins (IRAC MoA Group 6) are activators of the glutamate-gated chloride channels (GluCls) and cause neuronal and muscular system malfunctions (Lasota and Dybas 1991; Fisher and Mrozik 1992; IRAC 2019). They are acting on the gamma-aminobutyric acid (GABA) or glutamate receptors and cause abnormal chloride channel opening resulting in hyperexcitability and convulsions. Arena et al. (1995) demonstrated in insects that stimulation of glutamate (inhibitory) chloride channels is the most sensitive target site for the avermectins. The glutamate-gated chloride channels of insect and nematode skeletal muscle are especially important as they mediate avermectin-induced muscle paralysis in these organisms. These effects are mediated via a specific, high-affinity (10-10 M) binding site (Turner and Schaeffer 1989). To date, two representatives of this chemical group are registered for pest control; the insecticides abamectin and the emamectin benzoate. Emamectin benzoate is a second-generation avermectin insecticide and it is the benzoate salt forms of 4"-epi-methylami-no-4/1 deoxyavermectin B1 (Lasota and Dybas 1991; Fisher and Mrozik 1992; Lasota et al. 1996).

The insecticide emamectin benzoate is an extremely potent pest control tool for $T$. absoluta, resulting in high efficacy levels at the recommended label rate (Roditakis et al. 2013). In 2000, Siqueira et al. (2000b) reported resistance of $T$. absoluta to abamectin in populations from Brazil, however no other resistance cases involving avermectin have been detected since in South America. In year 2016, low resistance to emamectin benzoate reported for the first time in populations from Italy and Greece, however no cases of control failure have been detected (Roditakis et al. 2018). To date, additional reports for resistance to avermectins for T. absoluta from other regions of the world are practically absent. Enhanced metabolic detoxification has been reported in abamectin resistant pests, but it was not considered a major component of the resistance phenomenon (Rugg et al. 2005; Pu et al. 2010). The only fully documented case of biochemical resistance to avermectins has been reported in the two spotted spider mite Tetranychus urticae Koch, where Riga et al. (2014) demonstrated that the cytochrome P450 CYP392A16 is associated with high levels of abamectin resistance. Several mutations in GluCls, the target site of avermectins, are associated with resistance to this class of insecticides in major pests, however high fitness costs have been associated with abamectin resistant strains in some cases (Ishtiaq et al. 2014; Zaka et al. 2014; Afzal and Shad 2016). These reports involve the oriental leaf worm moth Spodoptera litura (F.) and the beet armyworm moth $S$. exigua (Hübner), suggesting that the advert effects of abamectin resistance could pose a limiting factor in resistance development in lepidoptera pests.

Hereby, emamectin benzoate resistance in T. absoluta was investigated, based on laboratory selection of a field strain that exhibited moderate susceptibility to the avermectin insecticide. Thereafter, comprehensive investigation of the resistance mechanisms involved was performed in an attempt to 
identify the genetic basis of emamectin benzoate resistance as well as the potential implications in pest management.

\section{Materials And Methods}

\subsection{Insect strains}

The T. absoluta population used in this study (GR-IER-16-6) was collected from infested greenhouse tomato crops (Solanum lycopersicum L.) in Greece during 2016. Details on the strain collection protocol are provided in Roditakis et al. (2018). The susceptible reference strain (ES-Sus) was collected in Spain in 2011 and was maintained under laboratory conditions without any exposure to insecticides. A detailed record for each population used in the study is provided on Table 1.

Each population was maintained in special incest proof rearing cages. For the development of the strains, 3 week old potted tomato plants (S. Iycopersicum, cv. Belladona) were used, which were maintained pestfree in large insect-proof containers under semi-field conditions. No insecticides were used during the plant development phase (for details see Roditakis et al. (Roditakis et al. 2013)). When adequate numbers of adult insects were available per cage, approximately 100 moths were collected and were allowed to oviposit on insect-free plants for 24 - 48h. Those plants were incubated separately until the larvae reached the second instar (L2). All rearing cages were maintained at $26 \pm 1^{\circ} \mathrm{C}, 65 \% \mathrm{RH}$ and $16 \mathrm{~h}$ light: $8 \mathrm{~h}$ dark photoperiod.

\subsection{Insecticides}

The following avermectin insecticide were used in the toxicological bioassays (commercial formulations): emamectin benzoate (Affirm ${ }^{\circledR} 095$ SG, Syngenta, UK) and abamectin (Doble $® 1.8 \mathrm{EC}$, Syngenta Crop Protection AG, Switzerland).

\subsection{Selection for emamectin benzoate resistance}

Selection of strain GR-IER-16-6 with emamectin benzoate was performed using foliar applied doses between $3 \mathrm{mg} \mathrm{L}^{-1}$ (first selection cycle) and up to $30 \mathrm{mg} \mathrm{L}^{-1}$. At least one thousand $2^{\text {nd }}$ instar larvae were used for each selection cycle. Strain GR-IER-16-6-S8 was obtained after eight sequential selection cycles.

\subsection{Toxicological bioassay method - Statistical analyses}

The IRAC method 022 (www.irac-online. org) was adopted for the toxicological bioassays. The method protocol is described in detail in Roditakis et al. (2013). Briefly, aqueous dispersions of commercial insecticide formulations were used in leaf dip bioassays. All bioassays were performed in a 32 cell repli dish (RT32W, Bioserve, US, www.insectrearing.com). Tomato leaflets, cut in square pieces were immersed in serial insecticide concentrations containing Triton X-100 $\left(0.2 \mathrm{~g} \mathrm{~L}^{-1}\right)$ as non-ionic wetting agent. Treated leaf pieces were allowed to dry for about $1 \mathrm{~h}$ and subsequently placed with their abaxial site on moist tissue paper in a multi-well repli-dish. A single second instar T. absoluta larva was carefully removed out 
of the galleries in infested tomato leaves, placed in each well and then the repli-dish was sealed with transparent ventilated adhesive lids. All treatments were conducted in a large insect rearing room with controlled environment $\left(26( \pm 1){ }^{\circ} \mathrm{C}, 50-60 \% \mathrm{RH}, 16 \mathrm{~h} \mathrm{~L}: 8 \mathrm{~h} \mathrm{D}\right)$. Larval mortality was assessed after $72 \mathrm{~h}$ of exposure. Mortality evaluations were performed with the aid of a light source and magnifying glass. A larva was considered dead if no movement could be observed. A larva was recorded as moribund if no coordinated movement or deficient response to external stimulus was observed (i.e. after gentle probing with a fine paint brush). The $\%$ mortality was expressed by combining the total number of dead and moribund insects.

Mortality data from all dose-response bioassays were subjected to probit analysis based on Finney (1964) using PriProbit 3.4 (Sakuma 1998). The software tests the linearity of dose-mortality response and provides the slope, the lethal concentrations (LC) and the $95 \%$ confidence limits (CL) of the lethal concentration for each mortality line. Using the appropriate function, the relative potency ratio among responses was calculated. Responses were considered significantly different when the 95\% confidence interval of relative potency ratio did not include the value 1. Percentage mortality values generated in bioassays was corrected using Abbott's formula (Abbott 1925). Results were compared to the reference strain to estimate the resistance ratio.

\subsection{Synergistic studies}

Synergists were used as tool to investigate the role of metabolic detoxification in emamectin benzoate resistance. For the synergism bioassays, second instar larvae of the emamectin benzoate selected GRIER-16-6-S8 strain, were exposed to sublethal concentrations of the synergists piperonyl butoxide (PBO, Sigma, UK), S,S,S tributyl phosphorotrithioate (DEF, Sigma, UK) and diethyl maleate (DEM, Sigma, UK), known to inhibit cytochrome P450 monooxygenases, esterases and glutathione S-transferases respectively. Exposure was achieved via tarsal contact on fresh dried synergist residues in coated glass vials (30 ml volume). For vial coating $300 \mu \mathrm{l}$ of acetonic solutions of PBO $\left(0.1 \mathrm{~g} \mathrm{~L}^{-1}\right)$, DEF $\left(0.1 \mathrm{~g} \mathrm{~L}^{-1}\right)$ or $\operatorname{DEM}\left(0.3 \mathrm{~g} \mathrm{~L}^{-1}\right)$ were added into each vial. Afterwards vials were placed horizontally on rotating metal rods for $1 \mathrm{~h}$. After rotation the vials were allowed to dry further for one more hour and then second instar larvae were placed into the vial and exposed to the synergist for 2 hours. Subsequently, the pre-exposed insects were used in bioassays with emamectin benzoate following IRAC method 022 as previously (2.3) described. The synergism ratio was calculated by dividing the $\mathrm{LC}_{50}$ - value of synergist-exposed larvae by the $\mathrm{LC}_{50}$ - value of non-exposed larvae.

\subsection{RNA isolation, RNAseq, cDNA synthesis}

In total, three different strains were included in the RNAseq experiment. 1) The laboratory susceptible strain ES-Sus was used as a reference strain, 2) the resistant strain 16-6-S which was selected with emamectin benzoate for eight consecutive generations and 3) its corresponding parental strain 16-6-P issued from the population GR-IER-16-6 which maintained in laboratory without selection pressure for 8 generations. RNA was extracted from a pool of twenty L2 larvae using the RNeasy mini kit (Qiagen). Four 
independent RNA extractions were included for each strain. The extracted RNA was further digested with Turbo DNase (Ambion) for the removal of genomic DNA contaminations. The integrity and the concentration of the RNA samples were assessed by Nanodrop spectrophotometer and by visualization on $1 \%$ agarose gel. RNA samples were sent to Macrogen (Seoul, Korea) for strand-specific, paired end sequencing using the Illumina HiSeq 2500 platform with $100 \mathrm{bp}$ per read. An aliquot of $1 \mu \mathrm{g}$ total RNA from each of the three aforementioned strains, served as a template for cDNA synthesis with Superscript III (Invitrogen, Carlsbad, CA, USA) using oligo-dT20, according to the manufacturer's instructions.

\subsection{Computational analyses (RNAseq)}

RNAseq reads from all three strains (total of 817.8 million reads) were assembled with Trinity v2.5.1 (Grabherr et al. 2011), using parameters "--seqType fq -SS_lib_type RF -max_memory 350G -CPU 20". InterProScan v5.28-67 (Jones et al. 2014) was used in order to identify conserved domains within each assembled transcript. Moreover, BLAST v2.8.0+ (Camacho et al. 2009) searches were run in order to identify similarities using the Uniref50 database (Suzek et al. 2015) that is specifically built for similaritybased functional annotation.

Transcript abundance was estimated with Kallisto (Bray et al. 2016). Next, the scripts bundled with Trinity were used for running the differential expression analysis with EdgeR (McCarthy et al. 2012) in order to find transcripts that were differentially expressed between the three populations (FDR <0.001). Custom Perl and bash scripts were used for parsing the EdgeR output and identifying genes of interest. Gene Ontology (GO) term analyses were done using gProfiler (Reimand et al. 2016).

In order to identify transcripts with similarity to cytochrome P450 (CYP) genes we first ran the TransDecoder program that is bundled with Trinity and obtained the encoded peptides in each transcript. Subsequently, putative CYP-related proteins were identified by the presence of the IPR001128 InterPro domain. Contaminating sequences were removed using the BLAST results against Uniref50; transcripts with a first hit not in lepidoptera or in any of its direct ancestors were treated as contaminants. The curated set of CYPs identified in the cotton bollworm H. armigera were obtained from Pearce et al. (2017) and used as a reference for classifying the T. absoluta P450s. Finally, the early-diverged CYP51A1 (Nelson 1999) from Homo sapiens was used as an outgroup. Multiple sequence alignment was performed with MAFFT v7.271 (Katoh and Standley 2013) with parameters "--auto -threads 8" and trimming was done with Trimal v1.2rev59 (Capella-Gutiérrez et al. 2009), with parameters "--gt 0.75". A Maximum Likelihood phylogeny with 100 bootstrap replicates was inferred with RAxML v8.2.11 (Stamatakis 2014), with parameters "-m PROTGAMMAAUTO". Branches with <50\% bootstrap support were collapsed with TreeGraph2 (Stöver and Müller 2010) and the resulting Newick tree was loaded to a locally deployed instance of EvolView v2 (He et al. 2016) for post-processing. The vector graphics editor Inkscape v0.92 was used for the final polishing.

2.8 Analysis of insecticide target polymorphism 
For the detection of polymorphisms in the gamma aminobutyric acid (GABA) receptor and the glutamategated chloride channel (GluCl) genes we firstly mapped the raw reads to the Trinity transcripts using hisat2 (Kim et al. 2015), then generated a mpileup file with samtools (Li et al. 2009), and searched for SNPs with VarScan v2.4.4 (Koboldt et al. 2012). Finally, the identified SNPs were visually inspected in the Integrative Genomics Viewer v2.8.4 (Robinson et al. 2011).

PCR amplification of GluCl and GABA gene fragments encompassing previously identified resistance mutations was also performed with primers listed in Supplementary file 1. PCR reactions (50ul) contained $1 \mu \mathrm{l} \mathrm{cDNA}, 0.2 \mu \mathrm{M}$ primers, $0.2 \mu \mathrm{M}$ dNTPs, $5 \mu \mathrm{L}$ of 10X Buffer and 1 U KAPA Taq DNA polymerase (KapaBiosystems). PCR was performed under the temperature cycling conditions of: 5 min at $95^{\circ} \mathrm{C}, 35$ cycles of $30 \mathrm{~s}$ at $95^{\circ} \mathrm{C}, 30 \mathrm{~s}$ at $57^{\circ} \mathrm{C}, 30 \mathrm{~s}$ at $72{ }^{\circ} \mathrm{C}$, followed by final extension of $2 \mathrm{~min}$ at $72^{\circ} \mathrm{C}$. PCR products were purified by using the nucleospin extract 2.0 kit (Macherey Nagel, Düren, Germany), according to manufacturer's instructions. Nucleotide sequences of purified PCR products were determined for both strands at CeMIA sequencing facility (CEMIA, SA., Greece). Obtained sequences were analysed with BioEdit v 7.0 (Hall 1999). The presence/absence of target site mutations was based on visual examination of sequencing chromatographs and RNAseq analysis.

\subsection{RNAseq validation by quantitive PCR}

P450s identified over-expressed among the studied strains were selected for validation using qPCR. Primers for the contigs of interest and a set of two reference genes (ribosomal protein RPL32A and elongation factor EFDelta) are listed in Supplementary file 1. RNA isolation, DNAse treatment and cDNA synthesis were described above (2.6). Quantitive PCR reactions were performed in quatriplate on QuantStudio 3 Real-Time PCR System (Applied biosystems) using 10ng of cDNA, $0.2 \mu \mathrm{M}$ primers and KAPA SYBR FAST qPCR Master Mix (KAPA BIOSYSTEMS) with the addition of ROX solution (KAPA BIOSYSTEMS). A 4-fold dilution series of pooled CDNA was used to access the efficiency of the qPCR reaction for each gene-specific primer pair. A no template control (NTC) was also included to detect possible contaminations. Experiments were performed using 4 biological and 2 technical replicates for each gene. A dissociation curve analysis was performed to check for the presence of a single amplicon. Relative expression levels were calculated according to Pfaffl (2001). Significant differences in gene expression were identified by pair-wise fixed reallocation randomization (Pfaffl et al. 2002).

\section{Results}

3.1 Selection, resistance and cross resistance to emamectin benzoate

Laboratory selections were conducted with emamectin benzoate on strain GR-IER-16-6 for eight sequential generations. Doses were adjusted to result in $60 \%-80 \%$ mortality in all cases, maintaining similar levels of selection pressure throughout. The probit analysis results are shown on Table 2 . The slope ranged between 0.73 and 1.48 indicating low level variability in the response of the tested strains. In all cases low chi-square values were observed indicating good fit of the probit model to the actual 
bioassay data. The estimated $\mathrm{LC}_{50}$ value for emamectin benzoate in the susceptible reference strain ESSus was at $0.09 \mathrm{mg} \mathrm{L}^{-1}$. The selected strain GR-IER-16-6-S8 exhibited LC $_{50} 5.46 \mathrm{mgL}^{-1}$, indicating a 60fold resistance ratio (RR) and a substantial 7-fold increase compared to the parental strain GR-IER-16-6 ( $\mathrm{LC}_{50} 0.81 \mathrm{mgL}^{-1}$, RR 9-fold, Table 2).

The cross-resistance pattern against the other registered avermectin insecticide, abamectin, was investigated (Table 2). The susceptible reference strain ES-Sus exhibited an $\mathrm{LC}_{50}$ at $0.05 \mathrm{mgL}^{-1}$, the field collected strain GR-IER-16-6 $0.24 \mathrm{mgL}^{-1}$ while the selected strain GR-IER-16-6-S8 $0.66 \mathrm{mgL}^{-1}$. The resistance ratio to abamectin increased after selection with emamectin benzoate from 4-fold to 13-fold, suggesting a moderate but clear cross resistance pattern between the two chemical compounds.

\subsection{Effect of synergists}

The probit analysis results from the synergistic bioassays are presented in Table 3. Pre-exposure to PBO resulted in significant reduction of $\mathrm{LC}_{50}$ levels to emamectin benzoate. More specifically, the $\mathrm{LC}_{50}$ was reduced from $5.46 \mathrm{mgL}^{-1}$ to $0.91 \mathrm{mgL}^{-1}$ after exposure to $\mathrm{PBO}$, resulting in a 6-fold synergism ratio indicating that P450s monooxygenases may play a key role in emamectin benzoate toxicity and/or resistance. Pre-exposure to DEM resulted in partially synergized emamectin benzoate resistance (3.8-fold synergism ratio). Analogous synergistic effects were not observed in the susceptible reference strain ESSus. When the esterase inhibitor DEF was used, a low synergistic ratio of 1.6-fold was observed, indicating no significant effects and minimal involvement of esterase EST in the phenotype (Table 3).

\subsection{Investigation of target site resistance mechanisms}

The glutamate-gated chloride channel (GluCl), the gamma amino butyric acid (GABA) gated chloride channel and the histamine-gated chloride channel (HisCl) interact with avermectins (Clark et al. 1995; Ludmerer et al. 2002; Wolstenholme and Rogers 2005; Prichard et al. 2012) although $\mathrm{GluCl}$ is considered the main target-site in arthropods (Sparks and Nauen 2015). Several point mutations in GluCl (G314D/G326E, I32IT) (Kwon et al. 2010; Dermauw et al. 2012; Wang et al. 2016a; Xue et al. 2020), and GABA (I281T, A301S/G/N, T305L, V332I, T350M and R357Q) reviewed by Feyereisen et al. (2015) have been previously tightly associated in arthropods with abamectin and cyclodien/phenylpyrazoles resistance respectively. Gene fragments of GluCl and GABA (transmembrane domains TM1-TM3) encompassing known SNPs were amplified and sequenced in order to investigate whether the presence of point mutations could explain the resistance phenotype. From the aforementioned mutations in all three strains the A301S mutation was identified in segregation, indicating that this mutation is unlikely to be associated with emamectin benzoate resistance in the studied strains. In all the other previously published positions all strains harbored the wild type allele (Figure 1). Additionally, the 36-bp deletion in $\mathrm{GluCl}$, previously associated with abamectin resistance in Plutella xylostella, was detected in all three strains, while a non-synonymous SNP at position 310 (E to V, T. absoluta numbering) was found in segregation and low frequency in GABA of the 16-6-P and the ES-Sus strains but not in the 16-6-S strain. Hence, their involvement in emamectin benzoate resistance is highly unlikely. 


\subsection{RNA sequencing}

Resistance of $T$. absoluta to emamectin benzoate was studied at the molecular level using RNA sequencing (RNAseq) on three populations of different resistance status; (a) the laboratory susceptible strain (ES-Sus), (b) the parental strain derived from the population GR-IER-16-6, which was maintained in the laboratory without emamectin benzoate selection pressure (16-6-P), and (c) the resistant strain selected with emamectin benzoate for eight consecutive generations (16-6-S) (Table 2). The Principal Components Analysis (PCA) revealed that $21.44 \%$ of the total variation could be explained by principal component 1 (PC1) while 16.91\% could be explained by PC2 (Supplementary File 2). The replicates from these three strains are clearly separated from each other, further confirming their quality and also the meaningfulness of downstream comparisons. It should be noted that replicates from populations 16-6-P and 16-6-S are only separated on PC2. In contrast, ES-Sus is obviously different from either 16-6-P or 166-S on both PC1 and PC2. Such finding is expected due to the fact that 16-6-S and 16-6-P share the same genetic background (originating from the same populations GR-IER-16-6, whereas the strain ES-Sus originates from Spain.

All RNAseq reads were pooled and assembled de novo with Trinity (Grabherr et al. 2011) into 549,601 transcripts $>200$ bp, originating from 233,453 unigenes. Running BUSCO (Waterhouse et al. 2018) on the transcriptome assembly showed that it is fairly complete, since it contains the complete sequence of $79.5 \%$ Insecta BUSCOs (Supplementary File 3). TransDecoder predicted proteins for 92,134 of the transcripts, with the majority of them having a significant hit against the Uniref50 database (Table 4). A set of unigenes was obtained after removing the following three types of proteins; (a) non-lepidopteran, contaminating sequences, (b) multiple isoforms of a unigene, and (c) proteins that were entirely contained within other, larger proteins. This final gene set included 32,502 proteins and, according to BUSCO, contained $75 \%$ of the Insecta BUSCO, only $4.3 \%$ of which was duplicated. An additional $16.5 \%$ of the BUSCOs were found as fragmented and $8.1 \%$ were missing (Supplementary File 4 ). As a result of this strict filtering scheme, the number of missing genes increased in the gene set. However, this was an acceptable trade-off in order to obtain a very clean unigene set.

A total of 143 P450s were identified in the aforementioned unigene set of $T$. absoluta. This number is higher than the $114 \mathrm{P} 450$ s previously identified in the genome of another lepidopteran insect, the cotton bollworm Helicoverpa armigera (Pearce et al. 2017). Such an increase is explained by the inclusion of P450 fragments in T. absoluta. We opted for including fragmented P450s because the present study has an exploratory nature and we aim at giving an exhaustive list of all candidate P450s. In this data set, representatives of all four major insect P450 clades were found. The majority of $T$. absoluta P450s (62 out of 143) belonged to Clan 4, 57 to Clan 3, 13 to the mitochondrial Clan, and 11 to Clan 2 (Table 5). The phylogenetic analysis allowed the classification of the T. absoluta P450s into clans and even into specific families, using the P450s from $H$. armigera as a reference (Figure 2). Moreover, it enabled the identification of P450s that are either duplicated or missing in T. absoluta, compared to $\mathrm{H}$. armigera. More specifically, it appears that there is at least one H. armigera P450 (CYP4CG14) that is duplicated in $T$. 
absoluta. Moreover, it is worth mentioning that no CYP18B1 ortholog was found in T. absoluta (Figure 2), most probably due to low expression levels.

\subsection{Differential expression summary}

Differential expression analysis on the entire set of 233,453 unigenes showed that 4,199 unigenes were significantly over-expressed $\left(\log _{2}|\mathrm{FC}|>2, \mathrm{FDR}<0.001\right)$ in the $16-6-\mathrm{S}$ strain (emamectin benzoate-selected) against either 16-6-P (parental), or ES-Sus (reference susceptible) (Supplementary File 5). Similarly, another 4,887 were significantly under-expressed in either one of the same comparisons (Supplementary File 6). This list includes two cytochrome P450s (DN75966_c4_g1, DN60698_c0_g2), two GSTs (DN60153_c3_g2, DN65736_c3_g1) and three ABC transporters (DN48209_c0_g2, DN52519_c0_g1, DN80907_c5_g2) (Table 6). It should be noted that the majority of these unigenes do not contain a protein-coding gene, according to the TransDecoder results (see above). More specifically, only 350 of the over-expressed unigenes contain a protein-coding gene, of which 290 have a significant similarity to another protein in the Uniref50 database. Similarly, there are 937 under-expressed unigenes that contain a predicted peptide, of which 859 have a significant similarity in Uniref50.

Among the 4,199 over-expressed unigenes there were 15 that (a) are commonly over-expressed in 16-6-S against either 16-6-P, or ES-Sus, (b) are full-length, and (c) have a significant hit in Uniref50 (Supplementary File 5). The majority of these genes are similar to uncharacterized proteins $(n=11)$, whereas the remaining four include a trypsin and a ribosomal protein. Using the same criteria we found 278 unigenes in the 4,887 under-expressed unigenes (Supplementary File 6).

Moreover, differential expression analysis revealed that two P450s were significantly over-expressed $\left(\log _{2}|\mathrm{FC}|>2, \mathrm{FDR}<0.001\right)$ in the 16-6-S resistant strain (Table 6). One of these genes (DN75966_c4_g1) is similar to CYP9G5 and therefore belongs to Clan 3, whereas the other (DN60698_c0_g2) is similar to CYP4AU1 and therefore belongs to Clan 4 (Figure 2). Despite an almost 32-fold over-expression for the DN75966_c4_g1 in the selected (16-6-S) compared to the parental one (16-6-P) strain, it should be noted that it is fragmented because the ORF encodes is only 113 amino acids (Table 6), In contrast, DN60698_c0_g2 appears to be full-length.

\subsection{RNAseq qPCR P450 validation}

Among the seven detoxification genes found over-expressed in the 16-6-S resistant strain we selected two P450 genes for qPCR validation; DN75966_c4_g1 and DN60698_c0_g2. The former was significantly over-expressed against the parental 16-6-P strain, whereas the latter was over-expressed compared to the Es-Sus strain. Quantitive PCR confirmed the levels of expression of DN60698_c0_g2 (log2FC=2.29, pvalue $<0.001)$ and supported the significant up-regulation calculated from the transcriptomic analysis $(\log 2 F C=2.212$, p-value <0.001; Table 6). In the contrary, DN75966_c4_g1 up-regulation in 16-6-S vs 16-6-P was not confirmed via qPCR.

\section{Discussion}


The tomato leaf miner exhibits extremely high capacity to develop resistance to wide range of insecticides. First resistance cases date back to early 2000 in South America (Siqueira et al. 2000a; Siqueira et al. 2000b; Lietti et al. 2005). However, the potency of the pest in resistance development was highlighted a few years later after its global invasion (Desneux et al. 2010). Resistance to several major classes of insecticides, including diamides, spinosyns, oxadiazines, pyrethrins and organophosphates have been reported over the past ten years while the pest was rapidly expanding to new areas worldwide (Biondi et al. 2018; Guedes et al. 2019).

Resistance monitoring is one of the main tools to evaluate the capacity of the pest to develop resistance to insecticides. The insecticide resistance of the tomato borer $T$. absoluta to insecticides has been monitored over the past years (Guedes and Picanço 2012; Campos et al. 2014; Silva et al. 2015; Yalcin et al. 2015; Silva et al. 2016a; Silva et al. 2016b; Roditakis et al. 2017; Grant et al. 2019) highlighting the trends in resistance development at a global scale. Such studies play a key role in evinced based crop protection and resistance management. An exception to the extreme resistance cases reported for $T$. absoluta is the class of avermectins.

In 2000, Siqueira et al. (2000b) reported up to 9.4 -fold resistance levels to abamectin in populations from Brazil; however, no other cases have been detected since. In these strains, efficacy of abamectin was synergized by piperonyl butoxide and triphenyl phosphate suggesting potential involvement of detoxification enzymes in abamectin resistance (Siqueira et al. 2001). The insecticide emamectin benzoate is an extremely potent pest control tool for $T$. absoluta. Although, it is extensively used in current IPM schemes, the resistance levels detected in general were low ( $>10$-fold) and no cases of control failure cases have reported in Europe to date (Roditakis et al. 2018). However, in year 2016, in three cases (Italy and Greece) resistance ratio reached 16 -fold, suggesting detection of low resistant levels and indicating a potential shift in the responses of $T$. absoluta populations to emamectin benzoate and development of incipient resistance to the chemical insecticide (Roditakis et al. 2018).

In other lepidopteran pests resistance studies to emamectin benzoate are limited or resistance is not fully defined. More specifically during an extensive survey in Pakistani (2005-2010) six S. litura populations exhibited low resistance levels (1.9-8.7-fold at $L_{50}$ and 4-10-fold at $L_{90}$ )(Ahmad and Mehmood 2015). Lethal effect of emamectin benzoate against the fourth-instar larvae of $S$. littoralis from Egypt was investigated and results indicated high susceptibility to the product but insecticide resistance was not evaluated (Kandil et al. 2020). There are reports for resistance development to $P$. xylostella in China (Wang and Wu 2014) but such studies involve mostly laboratory selection while extensive field surveys are missing. Resistance monitoring in US and Mexico for emamectin benzoate, dating back to 2006 indicated a resistance ratio <61-fold for $P$. xylostella (Zhao et al. 2006). More recently, regional surveys in Georgia and Florida, highlighted high efficacy levels of emamectin benzoate at the maximum level rate against $P$. xylostella, however resistance levels were not investigated (Riley et al. 2020). 
In this study, after 8 laboratory selection cycles with emamectin benzoate the $\mathrm{LC}_{50}$ increased to $5.46 \mathrm{mgL}^{-}$ 1 resulting in a 60- fold resistance ratio (RR). This is the highest resistance level ever reported for $T$. absoluta to emamectin benzoate, significantly higher to the respective RR values reported in Roditakis et al. (2018). However, compared to respective laboratory selection experiments conducted on T. absoluta with other chemical classes (examples indoxacarb, cyantraniliprole and spinosad), the observed 7-fold increase of the RR of the selected strain could be considerate as moderate. In other major pests, selection experiments with emamectin benzoate, resulted in higher RR values compared to this study. For example, selection with emamectin benzoate in $S$. exingua after inbreeding resulted in 1110-fold RR (Che et al. 2015). In a different study again with $S$. exingua, selection with emamectin benzoate for 6 generations resulted in increased RR by 526-fold (Ishtiaq et al. 2014). Laboratory selection of $P$. xylostella with abamectin resulted in a selected lab strain exhibiting 670-fold resistance (Wang and Wu 2014). One hypothesis suggested is that the $T$. absoluta population collected during the field surveys may not include genotypes that could exhibit high resistance levels to emamectin benzoate. On the other hand, $T$. absoluta is intensively treated for at least 10 years with avermectins and the absence of resistant genotypes may also indicate that the emamectin benzoate resistance is associated with reduced viability parameters in resistant individuals significantly restricting resistance development.

Cross resistance within the class of avermectins has been demonstrated in lepidopteran pests. The beet armyworm S. exingua exhibited cross resistance between emamectin benzoate and abamectin (Che et al. 2015). In the current study, selection with emamectin benzoate in T. absoluta resulted in analogous increase of resistance to abamectin suggesting potential cross resistance among the two avermectin insecticides however, abamectin resistance levels were moderate $(R R=13)$.

The synergist PBO strongly synergized emamectin benzoate and significantly reduced resistance levels in the selected strain (6-fold synergistic ratio, SR). It's indicative that the $\mathrm{LC}_{50}$ of the selected strain pretreated with $\mathrm{PBO}$ dropped at the $\mathrm{LC}_{50}$ levels of the parental strain. It is suggested that P450s could be one of the main mechanisms involved in emamectin benzoate resistance. The synergist DEM exhibited a moderate 3.8-fold synergistic effect and the difference in the responses were not statistically significant. In addition, the synergist DEF hand no effect on emamectin benzoate resistance. Based on these outputs EST and GSTs may have minimal involvement in emamectin benzoate resistance. Our results are in agreement with analogous prior studies on T. absoluta from Brazil, where PBO synergized abamectin toxicity (SR: 3- to 5-fold) in field collected populations (Siqueira et al. 2001). In an abamectin selected $P$. xylostella strain, all tested synergist exhibited no effect on abamectin toxicity (Wang et al. 2016a) however, in a different study by Pu et al. (2010) a 7.5-fold synergism with PBO was observed.

The glutamate-gated chloride channel ( $\mathrm{GluCl}$ ) and gamma amino butyric acid (GABA) interact with avermectins in insects and arthropods, causing abnormal chloride channel opening resulting in hyper excitability and convulsions. However, $\mathrm{GluCl}$ is considered as the main molecular target in arthropods. To date, no resistance mutations have been previously associated with $T$. absoluta resistance to avermectins. In P. xylostella a point mutation in the third transmembrane domain (TM3) of GluCl (A309V) 
has been strongly associated with abamectin resistance (Wang et al. 2016a), while a 36bp deletion was detected in higher frequency in an abamectin-selected strain (Liu et al. 2014). Additionally, G314D in GluCl1 and G326E, I321T in GluCl3 have been strongly correlated with abamectin resistance in $T$. urticae (Kwon et al. 2010; Dermauw et al. 2012; Xue et al. 2020). In the T. absoluta strains studied here we did not detected any of the aforementioned resistance mutations, but we detected the $36 \mathrm{bp}$ deletion, equally distributed among the three strains. Thus, it is highly unlikely for this deletion to be implicated in the observed resistance phenotype. Additionally, the presence of resistance mutations in GABA was also investigated. Several resistance mutations in GABA have been previously associated with resistance to cyclodiens and phenypirazoles in various arthropod species (reviewed by (Feyereisen et al. 2015)). In all studied strains we detected only the previously mentioned A301S substitution which was not fixed, as well as a novel non-synonymous SNP (E310V), in low frequency in the 16-6-P and the susceptible strain ES-Sus, but absent in the 16-6-S strain. Therefore it is also highly unlikely that these two mutations in GABA can be linked with emamectin benzoate resistance.

We compared gene expressions of the susceptible, the parental and the emamectin benzoate-selected strain and identified seven unigenes encoding for detoxification enzymes (2 P450s, 2 GSTs and 3 ABC transporters) that are overexpressed in the 16-6-S strain. The synergistic experiments combined with the absence of resistance mutations in target genes indicates the potential involvement of P450s in emamectin benzoate resistance. We focus on transcripts encoding for P450s since PBO caused significant reduction of emamectin benzoate toxicity and P450s are one of the most frequent players in insecticide metabolism (Dermauw et al. 2020). Detailed phylogenetic analysis revealed that P450 members of clan 3 (belonging to CYP9 family) and Clan 4 (belonging to CYP4 family) are overexpressed in the emamectin benzoate-selected strain (16-6-S). Clans 3 and 4 of CYP are the major arthropod CYP clans and the CYP4 and CYP9 families have been previously associated with insecticide resistance in several arthropods (Dermauw et al. 2020). To our knowledge, resistance to emamectin benzoate in $T$. absoluta and other lepidoptera species has not been analyzed at the gene level. Apart from P450s we also found other detoxification genes (GSTs and ABCs) that are overexpressed between the 16-6-S and the 16_6_P or ES_Sus. However, the involvement of GSTs and ABCs in emamectin benzoate resistant are not supported by our synergistic experiments since a) synergism with DEM, GST inhibitor, as not detected in the selected strain and $b$ ) the inhibitor of ABCs was not tested in the selected strain.

Combining data from RNAseq and qPCR, one P450 unigene (DN60698_c0_g2) was found significantly over-expressed in 16-6-S against ES-Sus. According to our phylogenetic analysis DN60698_c0_g2 is grouped with CYP4AU1 from $\mathrm{H}$. armigera and therefore belongs to Clan 4. P450 members of the CYP4 family have been previously associated with insecticide resistance in lepidoptera species such as Spodoptera sp (Huang et al. 2010; Wang et al. 2016b), P. xylostella (Baek et al. 2010). In non lepidopteran species the role of Clan 4 P450s in insecticide resistance has been functionally validated in vitro or in vivo in a number of species such as Diaphorina citri (Killiny et al. 2014), Laodelphax striatellus (Elzaki et al. 2015; Xiao et al. 2020), Locusta migratoria (Wu et al. 2020), Nilaparvata lugens (Zhang et al. 2016; Xu et al. 2020), Sogatella furcifera (Wang et al. 2019) and Leptinotarsa decemlineata (Kaplanoglu et al. 2017). Based on our transcriptomic and synergistic data, DN60698_c0_g2 qualifies as an excellent 
candidate for additional functional experiments, in order to elucidate its role in emamectin benzoate resistance.

Hereby, we present the first in-depth investigation of mechanisms involved in emamectin benzoate resistance in the tomato pest $T$. absoluta. Absence of mutations in the genes targeted by avermectins in combination with the synergistic action by PBO suggests the involvement of a metabolic detoxification mechanism. RNA sequencing and Qpcr confirmed the aforementioned hypothesis and identified the overexpression of one cytochrome P450 enzymes that could potentially be involved in emamectin benzoate metabolism. Moreover, the RNAseq were also analyzed in order to obtain a comprehensive set of $143 \mathrm{~T}$. absoluta cytochrome P450s that were further phylogenetically classified into one of the established clans. As a result, this study contributes significantly towards the rational resistance management of the most important tomato pest at a global level.

\section{Declarations}

Acknowledgments: This research is co-financed by Greece and the European Union (European Social Fund- ESF) through the Operational Programme "Human Resources Development, Education and Lifelong Learning" in the context of the project "Strengthening Human Resources Research Potential via Doctorate Research" (MIS-5000432), implemented by the State Scholarships Foundation (IKY) (M.S.). This research has been co-financed by Greek national funds through the Public Investments Project (PIP) of General Secretariat for Research \& Technology (GSRT), under the Emblematic Action "Research in the Agri-Food Sector of Crete", which is part of Subproject 2, "Pilot application of new standards of agricultural production" of the project sector of agri-food "(project code 2018 EE01300000). The research has been co-financed by the project "Smart Diagnostic tools and database to support precision plant protection in horticultural crops in Crete' 'SmartPP' funded by the Crete Operational Program 2014-2020 and co-funded by the European Regional Development Fund (ERDF), under the Priority Axis "Enhancing the Competitiveness, Innovation, and Entrepreneurship of Crete", Action 1.b.1: Demonstration - Experimental Development Projects, Promoting Research and Innovation in RIS3Crete. Also, this study is co-financed by Greece and the European Union (European Social Fund- ESF) through the Operational Programme «Human Resources Development, Education and Lifelong Learning» in the context of the project "Reinforcement of Postdoctoral Researchers - 2nd Cycle" (MIS-5033021), implemented by the State Scholarships Foundation (IKY) (A.I.).

Funding Fudging sources are stated in the Acknowledgements section

Conflicts of interest/Competing interests: Authors declare no conflicts of Interest

Availability of data and material The datasets used or analyzed during the current study are available from the corresponding author upon justified request. The sequencing reads are available from the Sequence Read Archive (SRA) under the bioproject accession PRJNA749726. https://dataview.ncbi.nlm.nih.gov/object/PRJNA749726? reviewer=qnecd 1 etje9fobbpfddb4s9vue 
Code availability Not applicable / No original software codes was used in this study

Author Contribution Statement: ER, JV conceived of and designed the experiments, MS conducted bioassay experiments, Al and PI analysed the RNAseq data and MS, Al, ER, JV wrote the manuscript.

Ethics approval This paper does not involve results of studies involving humans and/or animals.

Consent to participate All authors have agreed to participate in this manuscript

Consent for publication All authors have agreed to publish the information in this manuscript

\section{References}

1. Abbott WS (1925) A method of computing the effectiveness of an insecticide. J econ Entomol 18:265-267

2. Afzal MBS, Shad SA (2016) Characterization of Phenacoccus solenopsis (Tinsley)(Homoptera: Pseudococcidae) resistance to emamectin benzoate: cross-resistance patterns and fitness cost analysis. Neotrop Entomol 45:310-319

3. Ahmad M, Mehmood R (2015) Monitoring of resistance to new chemistry insecticides in Spodoptera litura (Lepidoptera: Noctuidae) in Pakistan. Journal of economic entomology 108:1279-1288

4. Arena JP, Liu KK, Paress PS, Frazier EG, Cully DF, Mrozik H, Schaeffer JM (1995) The mechanism of action of avermectins in Caenorhabditis elegans: correlation between activation of glutamatesensitive chloride current, membrane binding, and biological activity. The Journal of parasitology: 286-294

5. Baek JH, Clark JM, Lee SH (2010) Cross-strain comparison of cypermethrin-induced cytochrome P450 transcription under different induction conditions in diamondback moth. Pestic Biochem Physiol 96:43-50

6. Biondi A, Guedes RNC, Wan F-H, Desneux N (2018) Ecology, worldwide spread, and management of the invasive South American tomato pinworm, Tuta absoluta: past, present, and future. Annu Rev Entomol 63:239-258

7. Bray NL, Pimentel H, Melsted P, Pachter L (2016) Near-optimal probabilistic RNA-seq quantification. Nature biotechnology 34:525-527

8. Camacho C, Coulouris G, Avagyan V, Ma N, Papadopoulos J, Bealer K, Madden TL (2009) BLAST+: architecture and applications. BMC Bioinform 10:1-9

9. Campos MR, Silva TBM, Silva WM, Silva JE, Siqueira HAA (2014) Spinosyn resistance in the tomato borer Tuta absoluta (Meyrick) (Lepidoptera: Gelechiidae). J Pest Sci 88:405-412

10. Capella-Gutiérrez S, Silla-Martínez JM, Gabaldón T (2009) trimAl: a tool for automated alignment trimming in large-scale phylogenetic analyses. Bioinformatics 25:1972-1973

11. Che W, Huang J, Guan F, Wu Y, Yang Y (2015) Cross-resistance and inheritance of resistance to emamectin benzoate in Spodoptera exigua (Lepidoptera: Noctuidae). Journal of economic 
entomology 108:2015-2020

12. Clark JM, Scott JG, Campos F, Bloomquist JR (1995) Resistance to avermectins: extent, mechanisms, and management implications. Ann Rev Entomol 40:1-30

13. Dermauw W, llias A, Riga M, Tsagkarakou A, Grbić M, Tirry L, Van Leeuwen T, Vontas J (2012) The cys-loop ligand-gated ion channel gene family of Tetranychus urticae: implications for acaricide toxicology and a novel mutation associated with abamectin resistance. Insect Biochem Mol Biol 42:455-465

14. Dermauw W, Van Leeuwen T, Feyereisen R (2020) Diversity and evolution of the P450 family in arthropods. Insect Biochem Mol Biol 127:103490

15. Desneux N, Luna MG, Guillemaud T, Urbaneja A (2011) The invasive South American tomato pinworm, Tuta absoluta, continues to spread in Afro-Eurasia and beyond: The new threat to tomato world production. J Pest Sci 84:403-408

16. Desneux N, Wajnberg E, Wyckhuys KAG, Burgio G, Arpaia S, Narváez-Vasquez CA, González-Cabrera J, Ruescas DC, Tabone E, Frandon J (2010) Biological invasion of European tomato crops by Tuta absoluta: ecology, geographic expansion and prospects for biological control. J Pest Sci 83:197-215

17. Elzaki MEA, Zhang W, Han Z (2015) Cytochrome P450 CYP4DE1 and CYP6CW3v2 contribute to ethiprole resistance in Laodelphax striatellus (Fallén). Insect molecular biology 24:368-376

18. Feyereisen R, Dermauw W, Van Leeuwen T (2015) Genotype to phenotype, the molecular and physiological dimensions of resistance in arthropods. Pestic Biochem Physiol 121:61-77

19. Finney DJ (1964) A statistical treatment of the sigmoid response curve. Probit analysis 25

20. Fisher MH, Mrozik H (1992) The chemistry and pharmacology of avermectins. Annual review of pharmacology and toxicology (USA) 32: $537-53$

21. Grabherr MG, Haas BJ, Yassour M, Levin JZ, Thompson DA, Amit I, Adiconis X, Fan L, Raychowdhury $\mathrm{R}$, Zeng Q (2011) Full-length transcriptome assembly from RNA-Seq data without a reference genome. Nature biotechnology 29:644

22. Grant C, Jacobson R, llias A, Berger M, Vasakis E, Bielza P, Zimmer CT, Williamson MS, ffrenchConstant RH, Vontas J, Roditakis E, Bass C (2019) The evolution of multiple-insecticide resistance in UK populations of tomato leafminer, Tuta absoluta. Pest Manag Sci 75:2079-2085

23. Guedes RNC, Picanço MC (2012) The tomato borer Tuta absoluta in South America: Pest status, management and insecticide resistance. EPPO Bulletin 42:211-216

24. Guedes RNC, Roditakis E, Campos MR, Haddi K, Bielza P, Siqueira HAA, Tsagkarakou A, Vontas J, Nauen R (2019) Insecticide resistance in the tomato pinworm Tuta absoluta: patterns, spread, mechanisms, management and outlook. Journal of Pest Science: 1-14

25. Haddi K, Berger M, Bielza P, Rapisarda C, Williamson MS, Moores G, Bass C (2017) Mutation in the ace-1 gene of the tomato leaf miner (Tuta absoluta) associated with organophosphates resistance. $\mathrm{J}$ Appl Entomol 141:612-619 
26. Hall TA (1999) BioEdit: a user-friendly biological sequence alignment editor and analysis program for Windows 95/98/NT, pp. 95-98. [London]: Information Retrieval Ltd., c1979-c2000

27. He Z, Zhang H, Gao S, Lercher MJ, Chen W-H, Hu S (2016) Evolview v2: an online visualization and management tool for customized and annotated phylogenetic trees. Nucleic acids research 44:W236-W241

28. Huang S, Qin W, Chen Q (2010) Cloning and mRNA expression levels of cytochrome P450 genes CYP4M14 and CYP4S9 in the common cutworm Spodoptera litura (Fabricius). Scientia Agricultura Sinica 43:3115-3124

29. IRAC (2019) IRAC MoA Classification Scheme (Version 9.3). http://www.irac-online.org: (accessed, Jan. 2017)

30. Ishtiaq M, Razaq M, Saleem MA, Anjum F, ul Ane MN, Raza AM, Wright DJ (2014) Stability, crossresistance and fitness costs of resistance to emamectin benzoate in a re-selected field population of the beet armyworm, Spodoptera exigua (Lepidoptera: Noctuidae). Crop Prot 65:227-231

31. Jones P, Binns D, Chang H-Y, Fraser M, Li W, McAnulla C, McWilliam H, Maslen J, Mitchell A, Nuka G (2014) InterProScan 5: genome-scale protein function classification. Bioinformatics 30:1236-1240

32. Kandil MA, Fouad EA, El Hefny DE, Abdel-Mobdy YE (2020) Toxicity of Fipronil and Emamectin Benzoate and Their Mixtures Against Cotton Leafworm, Spodoptera littoralis (Lepidoptera: Noctuidae) With Relation to GABA Content. Journal of economic entomology 113:385-389

33. Kaplanoglu E, Chapman P, Scott IM, Donly C (2017) Overexpression of a cytochrome P450 and a UDP-glycosyltransferase is associated with imidacloprid resistance in the Colorado potato beetle, Leptinotarsa decemlineata. Scientific reports 7:1-10

34. Katoh K, Standley DM (2013) MAFFT multiple sequence alignment software version 7: improvements in performance and usability. Molecular biology evolution 30:772-780

35. Killiny N, Hajeri S, Tiwari S, Gowda S, Stelinski LL (2014) Double-stranded RNA uptake through topical application, mediates silencing of five CYP4 genes and suppresses insecticide resistance in Diaphorina citri. PloS one 9:e110536

36. Kim D, Langmead B, Salzberg SL (2015) HISAT: A fast spliced aligner with low memory requirements. Nat Methods 12:357-360

37. Koboldt DC, Zhang Q, Larson DE, Shen D, McLellan MD, Lin L, Miller CA, Mardis ER, Ding L, Wilson RK (2012) VarScan 2: somatic mutation and copy number alteration discovery in cancer by exome sequencing. Genome research 22:568-576

38. Kwon DH, Yoon KS, Clark JM, Lee SH (2010) A point mutation in a glutamate-gated chloride channel confers abamectin resistance in the two-spotted spider mite. Tetranychus urticae Koch Insect molecular biology 19:583-591

39. Lasota JA, Dybas RA (1991) Avermectins, a novel class of compounds: implications for use in arthropod pest control. Ann Rev Entomol 36:91-117

40. Lasota JA, Shelton AM, Bolognese JA, Dybas RA (1996) Toxicity of avermectins to diamondback moth (Lepidoptera: Plutellidae) populations: implications for susceptibility monitoring. Journal of 
economic entomology 89:33-38

41. Li H, Handsaker B, Wysoker A, Fennell T, Ruan J, Homer N, Marth G, Abecasis G, Durbin R, Genome Project Data Processing S (2009) The Sequence Alignment/Map format and SAMtools.

Bioinformatics 25:2078-2079

42. Lietti MMM, Botto E, Alzogaray RA (2005) Insecticide resistance in argentine populations of Tuta absoluta (Meyrick)(Lepidoptera: Gelechiidae). Neotropical Entomology 34:113-119

43. Liu F, Shi X, Liang Y, Wu Q, Xu B, Xie W, Wang S, Zhang Y, Liu N (2014) A 36-bp deletion in the alpha subunit of glutamate-gated chloride channel contributes to abamectin resistance in Plutella xylostella. Entomol Exp Appl 153:85-92

44. Ludmerer SW, Warren VA, Williams BS, Zheng Y, Hunt DC, Ayer MB, Wallace MA, Chaudhary AG, Egan MA, Meinke PT (2002) Ivermectin and nodulisporic acid receptors in Drosophila melanogaster contain both $\gamma$-aminobutyric acid-gated Rdl and glutamate-gated GluCla chloride channel subunits. Biochemistry 41:6548-6560

45. McCarthy DJ, Chen Y, Smyth GK (2012) Differential expression analysis of multifactor RNA-Seq experiments with respect to biological variation. Nucleic acids research 40:4288-4297

46. Nelson DR (1999) Cytochrome P450 and the individuality of species. Arch Biochem Biophys 369:110

47. Pearce SL, Clarke DF, East PD, Elfekih S, Gordon KHJ, Jermiin LS, McGaughran A, Oakeshott JG, Papanikolaou A, Perera OP (2017) Genomic innovations, transcriptional plasticity and gene loss underlying the evolution and divergence of two highly polyphagous and invasive Helicoverpa pest species. BMC Biol 15:1-30

48. Pfaffl MW (2001) A new mathematical model for relative quantification in real-time RT-PCR. Nucleic acids research 29:e45-e45

49. Pfaffl MW, Horgan GW, Dempfle L (2002) Relative expression software tool (REST@) for group-wise comparison and statistical analysis of relative expression results in real-time PCR. Nucleic acids research 30:e36-e36

50. Prichard R, Ménez C, Lespine A (2012) Moxidectin and the avermectins: consanguinity but not identity. International Journal for Parasitology: Drugs Drug Resistance 2:134-153

51. Pu X, Yang Y, Wu S, Wu Y (2010) Characterisation of abamectin resistance in a field-evolved multiresistant population of Plutella xylostella. Pest Management Science: Formerly Pesticide Science 66:371-378

52. Reimand J, Arak T, Adler P, Kolberg L, Reisberg S, Peterson H, Vilo J (2016) g: Profiler-a web server for functional interpretation of gene lists (2016 update). Nucleic acids research 44:W83-W89

53. Riga M, Tsakireli D, Ilias A, Morou E, Myridakis A, Stephanou EG, Nauen R, Dermauw W, Van Leeuwen T, Paine M (2014) Abamectin is metabolized by CYP392A16, a cytochrome P450 associated with high levels of acaricide resistance in Tetranychus urticae. Insect Biochem Mol Biol 46:43-53

54. Riley D, Smith H, Bennett J, Torrance P, Huffman E, Sparks A Jr, Gruver C, Dunn T, Champagne D (2020) Regional Survey of Diamondback Moth (Lepidoptera: Plutellidae) Response to Maximum 
Dosages of Insecticides in Georgia and Florida. Journal of economic entomology 113:2458-2464

55. Robinson JT, Thorvaldsdóttir H, Winckler W, Guttman M, Lander ES, Getz G, Mesirov JP (2011) Integrative genomics viewer. Nature biotechnology 29:24-26

56. Roditakis E, Mavridis K, Riga M, Vasakis E, Morou E, Rison JL, Vontas J (2017) Identification and detection of indoxacarb resistance mutations in the para sodium channel of the tomato leafminer, Tuta absoluta. Pest management science 73:1679-1688

57. Roditakis E, Skarmoutsou C, Staurakaki M (2013) Toxicity of insecticides to populations of tomato borer Tuta absoluta (Meyrick) from Greece. Pest management science 69:834-840

58. Roditakis E, Vasakis E, Garcia-Vidal L, del Rosario Martínez-Aguirre M, Rison JL, Haxaire-Lutun MO, Nauen R, Tsagkarakou A, Bielza P (2018) A four-year survey on insecticide resistance and likelihood of chemical control failure for tomato leaf miner Tuta absoluta in the European/Asian region. $\mathrm{J}$ Pest Sci 91:421-435

59. Roditakis E, Vasakis E, Grispou M, Stavrakaki M, Nauen R, Gravouil M, Bassi A (2015) First report of Tuta absoluta resistance to diamide insecticides. J Pest Sci 88:9-16

60. Rugg D, Buckingham SD, Sattelle DB, Jansson RK (2005) The insecticidal macrocyclic lactones

61. Sakuma M (1998) Probit analysis of preference data. Appl Entomol Zool 33:339-347

62. Silva GA, Picanço MC, Bacci L, Crespo ALB, Rosado JF, Guedes RNC (2011) Control failure likelihood and spatial dependence of insecticide resistance in the tomato pinworm, Tuta absoluta. Pest management science 67:913-920

63. Silva JE, Assis CPO, Ribeiro LMS, Siqueira HAA (2016a) Field-evolved resistance and cross-resistance of Brazilian Tuta absoluta (Lepidoptera: Gelechiidae) populations to diamide insecticides. Journal of economic entomology 109:2190-2195

64. Silva WM, Berger M, Bass C, Balbino VQ, Amaral MHP, Campos MR, Siqueira HAA (2015) Status of pyrethroid resistance and mechanisms in Brazilian populations of Tuta absoluta. Pestic Biochem Physiol 122:8-14

65. Silva WM, Berger M, Bass C, Williamson M, Moura DMN, Ribeiro LMS, Siqueira HAA (2016b) Mutation (G275E) of the nicotinic acetylcholine receptor a6 subunit is associated with high levels of resistance to spinosyns in Tuta absoluta (Meyrick)(Lepidoptera: Gelechiidae). Pestic Biochem Physiol 131:1-8

66. Siqueira HAA, Guedes RNC, Fragoso DdB, Magalhaes LC (2001) Abamectin resistance and synergism in Brazilian populations of Tuta absoluta (Meyrick)(Lepidoptera: Gelechiidae). International Journal of Pest Management 47:247-251

67. Siqueira HAA, Guedes RNC, Picanco MC (2000a) Cartap resistance and synergism in populations of Tuta absoluta (Lep., Gelechiidae). Journal of applied entomology 124:233-238

68. Siqueira HÁA, Guedes RNC, Picanço MC (2000b) Insecticide resistance in populations of Tuta absoluta (Lepidoptera: Gelechiidae). Agric For Entomol 2:147-153 
69. Sparks TC, Nauen R (2015) IRAC: Mode of action classification and insecticide resistance management. Pestic Biochem Physiol 121:122-128

70. Stamatakis A (2014) RAxML version 8: a tool for phylogenetic analysis and post-analysis of large phylogenies. Bioinformatics 30:1312-1313

71. Stöver BC, Müller KF (2010) TreeGraph 2: combining and visualizing evidence from different phylogenetic analyses. BMC Bioinform 11:1-9

72. Suzek BE, Wang Y, Huang H, McGarvey PB, Wu CH, UniProt C (2015) UniRef clusters: a comprehensive and scalable alternative for improving sequence similarity searches. Bioinformatics 31:926-932

73. Turner MJ, Schaeffer JM (1989) Mode of action of ivermectin. In: In Ivermectin and abamectin. Springer, pp 73-88

74. Urbaneja A, Vercher R, Navarro V, Porcuna JL, Garcia- Marí F (2007) La polilla del tomate, Tuta absoluta. Phytoma España 194:16-24

75. Wang R, Wu Y (2014) Dominant fitness costs of abamectin resistance in Plutella xylostella. Pest management science 70:1872-1876

76. Wang X-G, Ruan Y-W, Gong C-W, Xiang X, Xu X, Zhang Y-M, Shen L-T (2019) Transcriptome Analysis of Sogatella furcifera (Homoptera: Delphacidae) in Response to Sulfoxaflor and Functional Verification of Resistance-Related P450 Genes. Int J Mol Sci 20:4573

77. Wang X, Wang R, Yang Y, Wu S, O'Reilly AO, Wu Y (2016a) A point mutation in the glutamate-gated chloride channel of Plutella xylostella is associated with resistance to abamectin. Insect Mol Biol 25:116-125

78. Wang XG, Gao XW, Liang P, Shi XY, Song DL (2016b) Induction of cytochrome P450 activity by the interaction of chlorantraniliprole and sinigrin in the Spodoptera exigua (Lepidoptera: Noctuidae). Environ Entomol 45:500-507

79. Waterhouse RM, Seppey M, Simão FA, Manni M, loannidis P, Klioutchnikov G, Kriventseva EV, Zdobnov EM (2018) BUSCO applications from quality assessments to gene prediction and phylogenomics. Molecular biology evolution 35:543-548

80. Wolstenholme AJ, Rogers AT (2005) Glutamate-gated chloride channels and the mode of action of the avermectin/milbemycin anthelmintics. Parasitology 131:S85

81. Wu L, Zhang ZF, Yu Z, Yu R, Ma E, Fan YL, Liu TX, Feyereisen R, Zhu KY, Zhang J (2020) Both LmCYP4G genes function in decreasing cuticular penetration of insecticides in Locusta migratoria. Pest Manag Sci 76:3541-3550

82. Xiao Q, Deng L, Elzaki MEA, Zhu L, Xu Y, Han X, Wang C, Han Z, Wu M (2020) The Inducible CYP4C71 Can Metabolize Imidacloprid in Laodelphax striatellus (Hemiptera: Delphacidae). Journal of economic entomology 113:399-406

83. Xu L, Luo G, Sun Y, Huang S, Xu D, Xu G, Han Z, Gu Z, Zhang Y (2020) Multiple down-regulated cytochrome P450 monooxygenase genes contributed to synergistic interaction between chlorpyrifos and imidacloprid against Nilaparvata lugens. Journal of Asia-Pacific Entomology 23:44-50 
84. Xue W, Snoeck S, Njiru C, Inak E, Dermauw W, Van Leeuwen T (2020) Geographical distribution and molecular insights into abamectin and milbemectin cross-resistance in European field populations of Tetranychus urticae. Pest management science 76:2569-2581

85. Yalcin M, Mermer S, Kozaci LD, Turgut C (2015) Insecticide resistance in two populations of Tuta absoluta (Meyrick, 1917)(Lepidoptera: Gelechiidae) from Turkey. Türkiye Entomoloji Dergisi 39:137145

86. Zaka SM, Abbas N, Shad SA, Shah RM (2014) Effect of emamectin benzoate on life history traits and relative fitness of Spodoptera litura (Lepidoptera: Noctuidae). Phytoparasitica 42:493-501

87. Zhang Y, Yang Y, Sun H, Liu Z (2016) Metabolic imidacloprid resistance in the brown planthopper, Nilaparvata lugens, relies on multiple P450 enzymes. Insect Biochem Mol Biol 79:50-56

88. Zhao JZ, Collins HL, Li YX, Mau RFL, Thompson GD, Hertlein M, Andaloro JT, Boykin R, Shelton AM (2006) Monitoring of diamondback moth (Lepidoptera: Plutellidae) resistance to spinosad, indoxacarb, and emamectin benzoate. J Econ Entomol 99:176-181

\section{Tables}

Due to technical limitations, table 1 to 6 is only available as a download in the Supplemental Files section.

\section{Figures}


Px Glucl Tabs_Glucl ES-Sūs 16 . P Glucl 16_6_S_Glucl

Px Glucl Tabss Glucl ES-Sūs Glucl 16_6_P_Glucl 16_6_S_Glucl

B

Px_GABA Tabs GABA ES-Sus _GABA 16 6_S GABA 16 - ${ }^{-}$P_GABA

Px GABA Ta $\bar{b} s$ GABA ES-Sūs_GABA 16 6 S GABA 16 - 6 - ${ }^{-}-\mathrm{GABA}$

Px_GABA Tabs_GABA ES-Sus_GABA 16_6_S_GABA 16_6_P_GABA

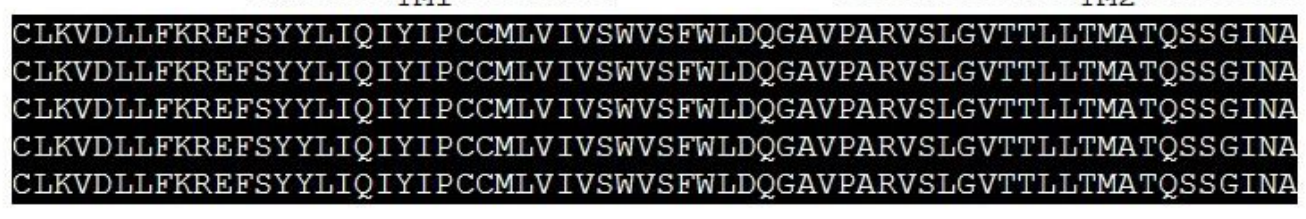

SLPPVSYTKA IDVWTGVCLTFVFGALLEFALVNYASRSDMHRENMKKTRREMEAAASMDAASDI SLPPVSYTKAIDVWTGVCLTFV FGALLEFALVNYASRSDMHRENMKKTRREMEAAA QMDAASDL SLPPVSYTKA IDVWTGVCLTFV FGALLEFALVNYASRSDMHRENMKKTRREMEAAA QMDAASDL SLPPVSYTKA IDVWTGVCLTEV FGALLEFALVNYASRSDMHRENMKKTRREMEAAAQMDAASDI SLPPVSYTKA IDVWTGVCLTFVFGALLEFALVNYASRSDMHRENMKKTRREMEAAAQMDAASDL है్ㅏ वे

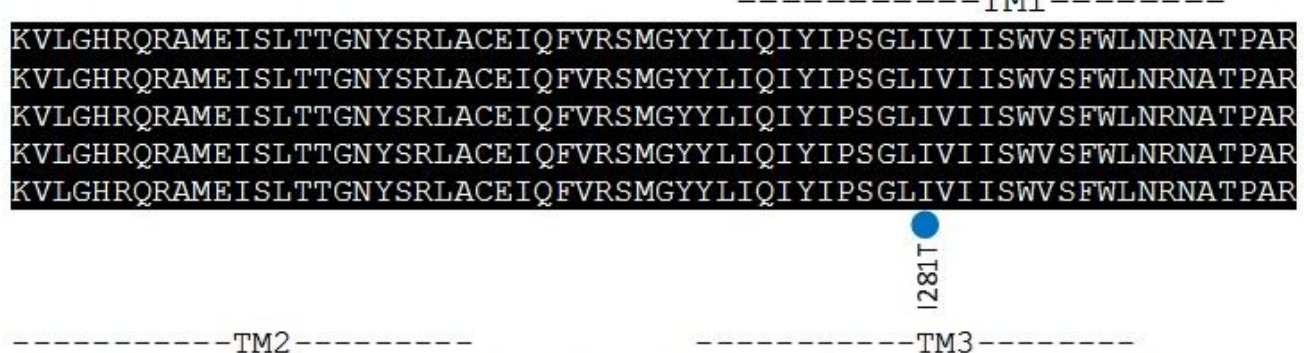

VALGVTTVLTMTTLMSSTNAAL PKISYVKS I DVYLGTCFVMVFASLLEYATVGYMAKRIQMRKQ VSLGVTTVLTMTTLMSSTNAAL PKISYVKS I DVYLGTCFVMVFASLLEYATVGYMAKRIQMRKQ VSLGVTTVLTMTTLMSSTNAALPKISYVKSI DVYLGTCFVMVFASLLEYATVGYMAKRIQMRKQ V SLGVTTVLTMTTLMSSTNAAL PKIS YVKS I DVYLGTCFVMVFASLLEYATVGYMAKRIQMRKQ VSLGVTTVLTMTTLMSSTNAALPKIS YVKS I DVYLGTCFVMVFASLLEYATVGYMAKRIQMRKQ 总
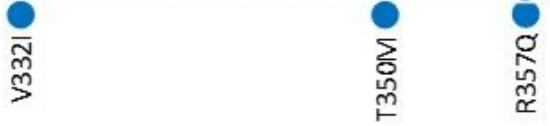

RFVAMQKIASE $Q K K L P P D C P P L G D P H T L S K M G T$ RFVAIQKIASEKKMPPLDCPPVGDPHTLSKMST RFVAIQKIASEKKMPPLDCPPVGDPHTLSKMST RFVAIQKIASEKKMPPLDCPPVGDPHTLSKMST RFVAIQKIASEKKMPPLDCPPVGDPHTLSKMST

\section{Figure 1}

Aminoacid Alignment of the transmembrane regions 1-3 (TM1-TM3) of Tuta absoluta strains (ES-Sus, 16_6_S and 16_6_P) for GluCl (A) and GABA (B) gene. P. xylostella A309V and T. urticae I321T and G314D resistance mutations, in $\mathrm{GluCl}$ gene associated with abamectin resistance, are indicated with red triangle and green circles respectively. Re-sistance mutations in GABA genes associated with cyclodien/phenylpyrazoles resistance in arthropods are indicated by blue circles, while aminoacid position A301S detected in segre-gation in all T. absoluta strains is indicated in red font. 


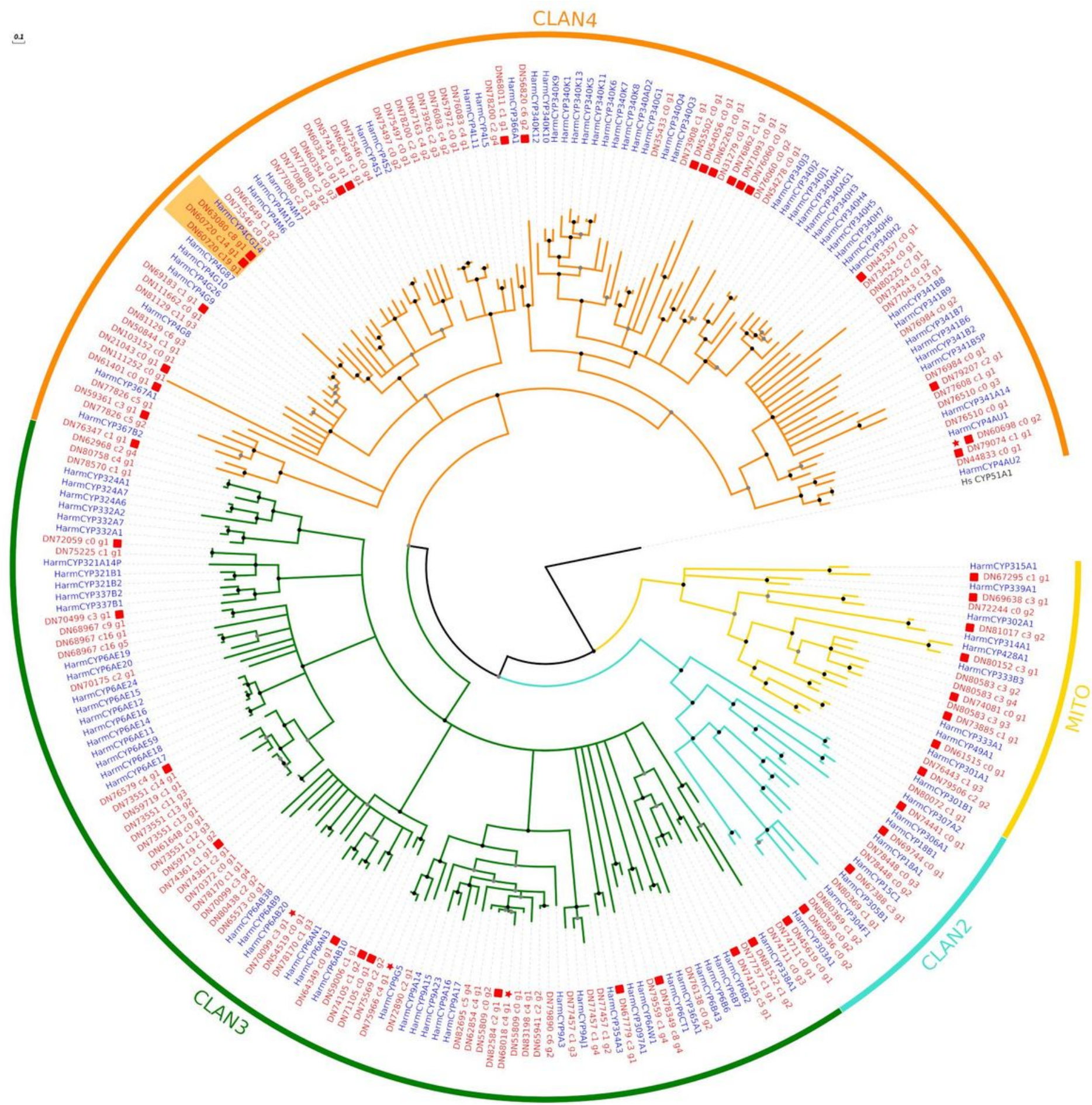

Figure 2

Phylogeny of all identified T. absoluta CYPs (names in red), compared to those previously identified in $\mathrm{H}$. armigera (names in blue). This comparison allowed for the classification of T. absoluta CYPs, using $\mathrm{H}$. armigera as a reference. CYPs marked with a star denote CYPs that are significantly up-regulated in a resistant population compared to a more susceptible one $(p<0.001, \log 2 \mathrm{FC}>2)$. CYPs marked with a square denote nearly full-length unigenes ( $>400$ amino acids). Bootstrap support is shown with dots; black for $>75 \%$ and grey for $50-75 \%$. Nodes with a support $<50 \%$ were collapsed. 


\section{Supplementary Files}

This is a list of supplementary files associated with this preprint. Click to download.

- Tables.docx

- SupplementaryFile.docx 